\title{
High-Throughput and Rapid Screening of Low-Mass Hazardous Compounds in Complex Samples
}

\author{
Jing Wang, ${ }^{\dagger, \dagger}$ Qian Liu, ${ }^{* \dagger}{ }^{\dagger}$ Yan Gao, ${ }^{\dagger}$ Yawei Wang, ${ }^{\dagger}$ Liangqia Guo, $^{\ddagger}$ and Guibin Jiang ${ }^{\dagger}$ \\ ${ }^{\dagger}$ State Key Laboratory of Environmental Chemistry and Ecotoxicology, Research Center for Eco-Environmental Sciences, Chinese \\ Academy of Sciences, Beijing 100085, China \\ ${ }^{\ddagger}$ Department of Chemistry, Fuzhou University, Fuzhou 350002, China
}

Supporting Information

ABSTRACT: Rapid screening and identification of hazardous chemicals in complex samples is of extreme importance for public safety and environmental health studies. In this work, we report a new method for high-throughput, sensitive, and rapid screening of low-mass hazardous compounds in complex media without complicated sample preparation procedures. This method is achieved based on size-selective enrichment on ordered mesoporous carbon followed by matrix-assisted laser desorption/ionization-time-of-flight mass spectrometry analysis with graphene as a matrix. The ordered mesoporous carbon CMK-8 can exclude interferences from large molecules in complex samples (e.g., human serum, urine, and environ-

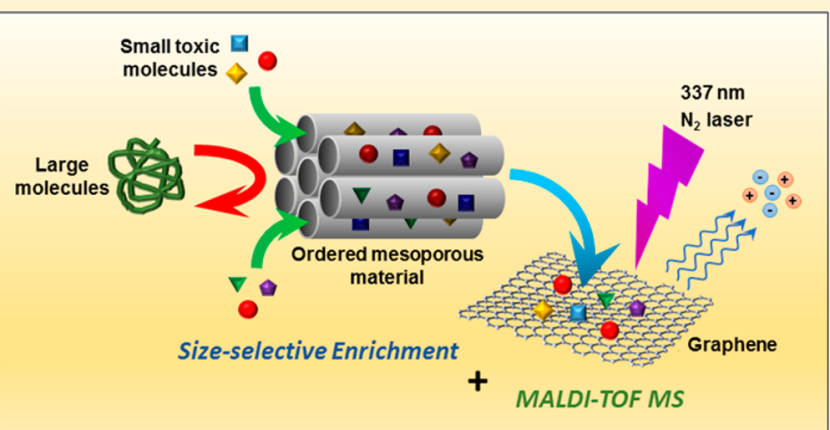
mental water samples) and efficiently enrich a wide variety of low-mass hazardous compounds. The method can work at very low concentrations down to part per trillion (ppt) levels, and it is much faster and more facile than conventional methods. It was successfully applied to rapidly screen and identify unknown toxic substances such as perfluorochemicals in human serum samples from athletes and workers. Therefore, this method not only can sensitively detect target compounds but also can identify unknown hazardous compounds in complex media.

Sc creening and identification of hazardous chemicals in complex samples such as human body fluids and environmental media is of extreme importance for public safety and environmental health studies. A large fraction of hazardous chemicals are low-mass compounds $\left(M_{\mathrm{w}}<1000\right.$ $\mathrm{Da})$. However, analysis of low-mass compounds in complex samples is still a great challenge due to (1) extremely low concentrations (most of small toxic molecules are present in real samples at extremely low concentrations (parts per billion or even parts per trillion levels), ${ }^{1,2}$ and there still is a lack of reliable methods capable of high-throughput screening and identifying of these compounds at such low levels) and (2) interferences from sample matrices. The interferences from complex sample matrices (e.g., large molecules and salts) can greatly suppress the analytical responses of the target small molecules.

Conventional methods for analysis of low-mass compounds in complex samples mainly rely on gas or high-performance liquid chromatography (GC or HPLC) coupled to mass spectrometry (MS) or spectrophotometry detectors. ${ }^{3}$ These methods are low throughput and require complicated sample preparation procedures that are time-consuming and laborious. Chip-based techniques can provide high-throughput analysis for target compounds, but they also suffer from complex sample matrices and lack accurate identification ability. ${ }^{4}$ Matrix-assisted laser desorption/ionization-time-of-flight mass spectrometry
(MALDI-TOF MS) provides a powerful tool for highthroughput analysis of large molecules (e.g., proteins, peptides, and polymers, etc.), but its application to small molecules is seriously hindered by insufficient sensitivity, ionization suppression effect, and matrix interferences in low-mass regions.

Here we report a method for high-throughput and rapid screening of low-mass hazardous compounds in complex media based on size-selective enrichment on ordered mesoporous material followed by MALDI-TOF MS analysis (Figure 1). Ordered mesoporous materials refer to materials containing ordered pores with diameters in the range of $2-50 \mathrm{~nm} .^{5,6}$ Due to the molecular sieving effect, ${ }^{5,6}$ ordered mesoporous materials can selectively enrich low-mass compounds from complex samples meanwhile excluding the interferences from large molecules. Furthermore, the ultralarge surface area of ordered mesoporous materials can offer high capacity for the enrichment. Thus, the use of ordered mesoporous materials was expected to solve both the problems of insufficient analytical sensitivity and matrix interferences from large molecules. Although ordered mesoporous materials have been previously used to separate different-sized biopolymers, ${ }^{7-11}$ to the best of

Received: April 24, 2015

Accepted: June 3, 2015

Published: June 3, 2015 


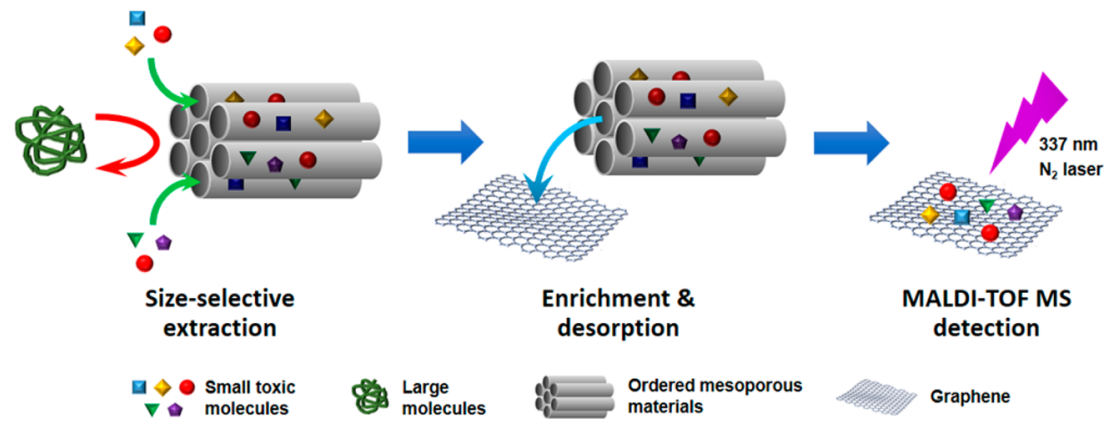

Figure 1. Scheme showing the procedures for the screening of low-mass toxic compounds.

our knowledge, their application in size-selective enrichment and analysis of small molecules is hitherto unreported. MALDITOF MS provides the ability of high-throughput identification and detection of multiple compounds in a single run. Graphene, a carbon material with a two-dimensional and oneatom-thick structure, ${ }^{12,13}$ was used as a matrix in MALDI to offer high LDI efficiency and reduce background interferences in a low-mass region due to its strong optical-absorption properties and efficient electron-phonon coupling. ${ }^{14-18}$ This method does not require any complicated sample preparation procedures and can be applied to rapidly screen and identify a wide variety of trace hazardous compounds in diverse complex samples.

\section{EXPERIMENTAL SECTION}

Chemicals and Materials. The ordered mesoporous carbon (CMK-3 and CMK-8), ordered mesoporous silica SBA-15, and chemically converted graphene were purchased from XFNANO Materials Tech Co. (Nanjing, China). The ordered mesoporous silica MCM-41 was purchased from JCNano Technology Co. (Nanjing, China). Bisphenol S (BPS), tetradecyldimethylbenzylammonium chloride hydrate (TDBAC), and tetrabromobisphenol A (TBBPA) were from TCI (Tokyo, Japan). 2,2',4,4'-Tetrabromodiphenyl ether (BDE-47) was purchased from Accustandard (New Haven, CT, USA). Arsenic(III) standard at $1000 \mu \mathrm{g} / \mathrm{mL}$ in $0.5 \%(\mathrm{v} / \mathrm{v})$ $\mathrm{HCl} / 0.3 \%$ (w/v) $\mathrm{NaOH} / 0.06 \%$ (w/v) $\mathrm{NaHCO}_{3}$ was purchased from Inorganic Ventures (Christiansburg, VA, USA). Pentachlorophenol (PCP) and estradiol (E2) were from Dr. Ehrenstorfer (Augsburg, Germany). Perfluorooctanesulfonate (PFOS), hexadecyldimethylbenzylammonium chloride (HDBAC), dodecyldimethylbenzylammonium chloride (DDBAC), cetyltrimethylammonium bromide (CTAB), tetradecyltrimethylammonium bromide (TTAB), and bovine serum albumin (BSA) were bought from Sigma (St. Louis, MO, USA). Humic acid (HA) was purchased from Alfa Aesar (Ward Hill, MA, USA). Dichloromethane (DCM) and acetone of HPLC grade were from J. T. Baker (Phillipsburg, NJ, USA). Ultrapure water from a Millipore Milli-Q system (Billerica, MA, USA) was used throughout. All reagents were of analytical grade unless otherwise noted.

Synthesis of Acid-Oxidized Graphene. A nitric acidoxidized graphene (AOG) was used as a MALDI matrix in this study and synthesized as described previously. ${ }^{17}$ Briefly, the chemically converted graphene was refluxed in $2 \mathrm{M} \mathrm{HNO}_{3}$ (Merck, Darmstadt, Germany) at $105{ }^{\circ} \mathrm{C}$ for $24 \mathrm{~h}$. The solid product was washed with water several times and then dispersed in water at $1 \mathrm{mg} / \mathrm{mL}$ by the aid of sonication for 2 $h$ to give a stable aqueous suspension.
Enrichment of Target Chemicals. The ordered mesoporous materials were dispersed in water at a concentration of $30 \mathrm{mg} / \mathrm{mL}$ by the aid of sonication before use. To enrich the target compounds, $400 \mu \mathrm{L}$ of the ordered mesoporous material dispersion was added to $10 \mathrm{~mL}$ of sample solution. The mixture was shaken at room temperature for $1 \mathrm{~h}$ and then centrifugated at $4000 \mathrm{rpm}$ for $15 \mathrm{~min}$. The precipitates were collected and mixed with $3 \mathrm{~mL}$ of $1: 1(\mathrm{v} / \mathrm{v})$ DCM/acetone to elute the analytes. The mixture was sonicated for $30 \mathrm{~min}$ and centrifugated at $9000 \mathrm{rpm}$ for $15 \mathrm{~min}$. The supernatant was transferred to a new tube and concentrated to $20 \mu \mathrm{L}$ under a gentle steam of $\mathrm{N}_{2}$ for MALDI-TOF MS analysis.

MALDI-TOF MS Analysis. MALDI-TOF MS measurements were performed on a Bruker Daltonics Autoflex III Smartbean MALDI-TOF mass spectrometer in reflectron mode controlled by a FlexControl software. A $337 \mathrm{~nm}$ nitrogen laser with the frequency of $100 \mathrm{~Hz}$ was used. The spectra were recorded by summing 200 laser shots. The AOG was used as a MALDI matrix. ${ }^{17}$ To perform the MS analysis, $1 \mu \mathrm{L}$ of the sample solution was mixed with $3 \mu \mathrm{L}$ of AOG dispersion on a stainless steel MTP target frame III (Bruker Daltonics) followd by air-drying. The data processing was performed with the FlexAnalysis 3.0 software.

Real Sample Analysis. The human serum and urine samples from healthy male athletes were kindly provided by the National Institute of Sports Medicine of China (Beijing, China) and stored at $-20{ }^{\circ} \mathrm{C}$. River water samples were collected from Xiaoling River (Beijing, China). The collected water samples were filtered through a $0.45 \mu \mathrm{m}$ Millipore PTFE membrane immediately after sampling and stored in amber glass bottles at $4{ }^{\circ} \mathrm{C}$. The worker serum samples were collected from 30 workers in a perfluorochemical plant (Wuhan, China) and stored at $-20{ }^{\circ} \mathrm{C}$.

For human serum samples, $50 \mu \mathrm{L}$ of the serum samples were diluted in $1.5 \mathrm{~mL}$ of water and then subjected to the extraction and MS analysis. For urine samples, $1 \mathrm{~mL}$ of the urine samples was diluted to $10 \mathrm{~mL}$ with water. The river water samples were directly analyzed without dilution.

\section{RESULT AND DISCUSSION}

Selection of Enrichment Material. To demonstrate our concept as depicted in Figure 1, we first selected a suitable ordered mesoporous material for the size-selective enrichment. We have tested two mesoporous carbons (CMK-3 and CMK8) and two mesoporous silicas (SBA-15 and MCM-41). TEM measurements show that these materials had uniform narrow pores (Figure 2) with very high specific surface areas $(>500$ $\mathrm{m}^{2} / \mathrm{g}$ ) and nanoscaled pore sizes (Table 1 ). The $\mathrm{N}_{2}$ sorption isotherms and pore distribution curves of the ordered 

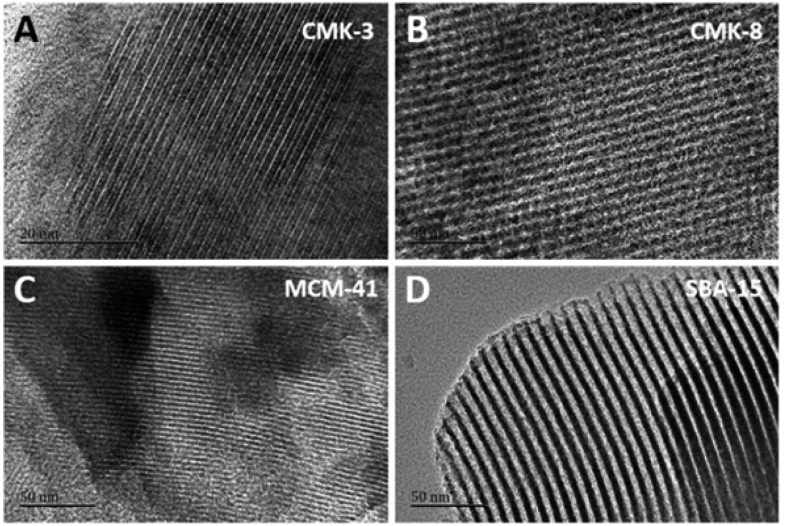

Figure 2. TEM images of the ordered mesoporous materials tested in this study.

Table 1. Specific Surface Area and Average Pore Size of Ordered Mesoporous Materials Obtained by Using the Brunauer-Emmett-Teller (BET) Method

$\begin{array}{lcc}\text { material } & \text { specific surface area }\left(\mathrm{m}^{2} / \mathrm{g}\right) & \text { av pore size }(\mathrm{nm}) \\ \text { CMK-3 } & 924.9 & 4.88 \\ \text { CMK-8 } & 536.9 & 6.30 \\ \text { MCM-41 } & 862.5 & 4.67 \\ \text { SBA-15 } & 600.4 & 6.47\end{array}$

mesoporous materials are given in Figure S1 in the Supporting Information. Various types of low-mass hazardous compounds were selected as model analytes to test the universality of the method, including a perfluorochemical (PFOS), brominated flame retardants (BDE-47 and TBBPA), an endocrine disrupter (E2), an organochlorine pesticide (PCP), an industrial additive (BPS), linear surfactants ( $\mathrm{CTAB}$ and $\mathrm{TTAB}$ ), aromatic surfactants (HDBAC, TDBAC, and DDBAC), and inorganic As ${ }^{\text {III }}$. These compounds are widely used in industrial or consumer products but have been demonstrated to be harmful to human health or pose potential risks to ecosystems (see Supporting Information for detailed reasons for selection). ${ }^{1,2}$ The MALDI-TOF MS was performed in both negative ion and positive ion mode to ensure that all target compounds could be detected in a single run (Table 2). The graphene matrix was mildly oxidized by diluted nitric acid to improve its dispersibility in water and on the MALDI target (Supporting Information Figure S2). ${ }^{17}$

We used two types of large molecules, BSA $\left(M_{\mathrm{w}} 66.4 \mathrm{kDa}, 14\right.$ $\mathrm{nm} \times 4 \mathrm{~nm} \times 4 \mathrm{~nm})$ and HA, as mimics of biological and environmental matrices to examine the matrix interferences from large molecules in MS analysis. We found that the

Table 2. Feature Peaks and LODs of Typical Low-Mass Hazardous Compounds

\begin{tabular}{lllllll}
\multicolumn{3}{c}{ negative ion mode } & & \multicolumn{3}{c}{ positive ion mode } \\
\cline { 1 - 3 } \cline { 5 - 6 } compound & $m / z$ & $\begin{array}{c}\text { LOD } \\
(\mathrm{pg} / \mathrm{mL})\end{array}$ & & compound & $m / z$ & $\begin{array}{c}\text { LOD } \\
(\mathrm{pg} / \mathrm{mL})\end{array}$ \\
PFOS & 498.5 & 10 & & As ${ }^{\mathrm{III}}$ & 151.4 & $5 \times 10^{4}$ \\
TBBPA & 542.4 & $1 \times 10^{4}$ & & CTAB & 283.8 & 10 \\
E2 & 270.5 & 50 & & TTAB & 255.8 & 10 \\
BDE-47 & 250.1 & 0.5 & & HDBAC & 359.9 & 50 \\
BPS & 248.3 & 100 & & TDBAC & 331.9 & 50 \\
PCP & 264.2 & 0.1 & & DDBAC & 303.8 & 50
\end{tabular}

presence of BSA or HA in a sample could indeed seriously interfere with the MS detection of small molecules (Supporting Information Figures S3 and S4). Even when the samples contained only $10 \mu \mathrm{g} / \mathrm{mL}$ BSA or HA, the MS signals of small molecules could be greatly decreased or even totally lost. The possible reason is that large molecules have a stronger absorption for the laser energy than small molecules and can interfere with the ionization of small molecules in MALDI.

Figure 3 compares the performance of different ordered mesoporous materials in size-selective enrichment of low-mass
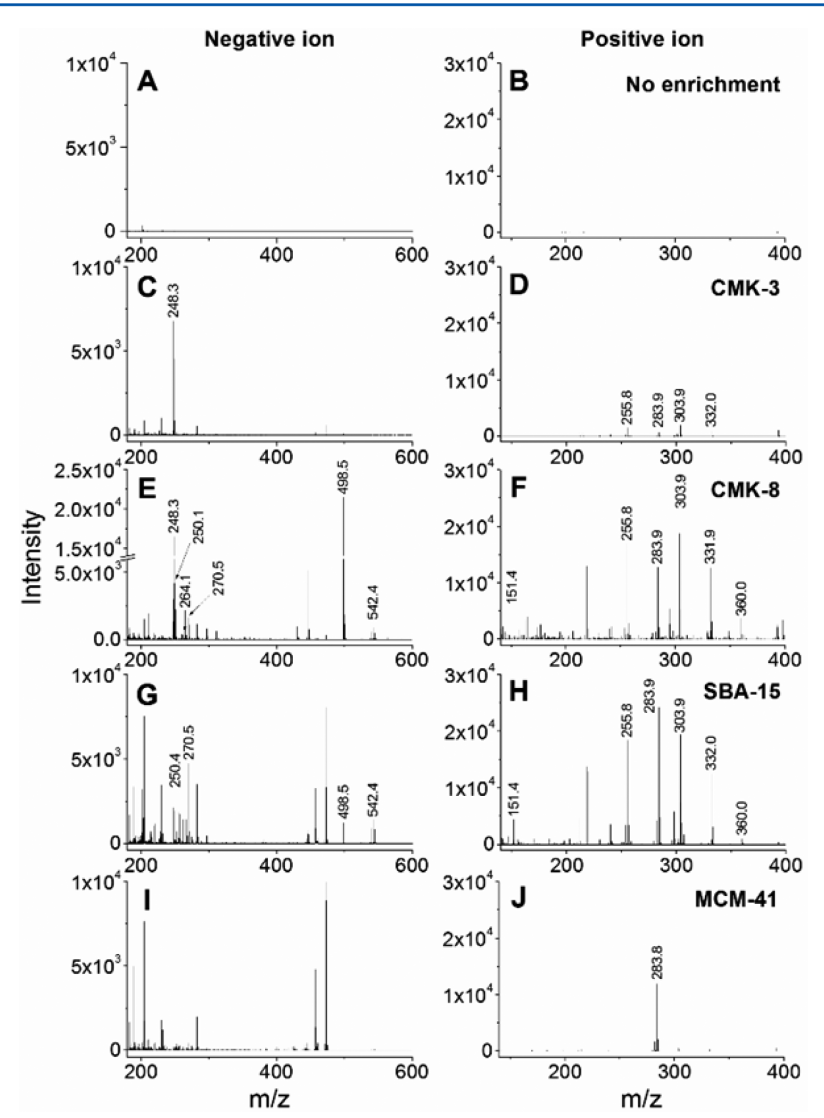

Figure 3. Comparison of different ordered mesoporous materials in size-selective enrichment of typical low-mass compounds in the presence of $10 \mu \mathrm{g} / \mathrm{mL}$ BSA: Direct analysis (A, B) without enrichment, and with enrichment (C, D) using CMK-3, (E, F) using CMK-8, (G, H) using SBA-15, and (I, J) using MCM-41. The MALDI-TOF MS was performed in both negative ion (left column) and positive ion mode (right column) using graphene as a matrix. Analyte concentrations: PFOS, $10 \mathrm{ng} / \mathrm{mL}$; BPS, $100 \mathrm{ng} / \mathrm{mL}$; PCP, 100 $\mathrm{ng} / \mathrm{mL}$; TBBPA, $100 \mathrm{ng} / \mathrm{mL}$; E2, $500 \mathrm{ng} / \mathrm{mL}$; BDE-47, $500 \mathrm{ng} / \mathrm{mL}$; As ${ }^{\text {III }}, 150 \mathrm{ng} / \mathrm{mL}$; HDBAC, $50 \mathrm{ng} / \mathrm{mL}$; TDBAC, $50 \mathrm{ng} / \mathrm{mL} ;$ DDBAC, $50 \mathrm{ng} / \mathrm{mL}$; CTAB, $10 \mathrm{ng} / \mathrm{mL}$; TTAB, $10 \mathrm{ng} / \mathrm{mL}$. The $m / z$ values for the features peaks of the target compounds are labeled in the spectra.

compounds in the presence of $10 \mu \mathrm{g} / \mathrm{mL}$ BSA. Without enrichment no feature peaks of the target compounds were observed (Figure 3A,B). After enrichment by CMK-3, peaks of $[\mathrm{M}-\mathrm{H}]^{-}$of BPS at $\mathrm{m} / z 248.3$ in negative ion mode and $[\mathrm{M}-$ $\mathrm{Br}]^{+}$of surfactants (TTAB, CTAB, DDBAC, and TDBAC) at $m / z 255.8,283.9,303.9$, and 332.0 in positive ion mode could be detected, but the intensities were low (Figure 3C,D). The best performance was achieved when using CMK-8 as a sorbent, with which all the tested compounds could be detected with high peak intensities (Figure 3E,F). In addition to the peaks mentioned previously, we also obtained peaks of $[\mathrm{M}-$ 
$\left.\mathrm{C}_{6} \mathrm{H}_{3} \mathrm{Br}_{2}\right]^{-}$of BDE-47 and $[\mathrm{M}-\mathrm{H}]^{-}$of PCP, E2, PFOS, TBBPA at $m / z 250.1,264.1,270.5,498.5$, and 542.4 in negative ion mode and $[\mathrm{M}-\mathrm{H}+2 \mathrm{Na}]^{+}$of $\mathrm{As}^{\mathrm{III}}$ and $[\mathrm{M}-\mathrm{Br}]^{+}$of HDBAC at $m / z 151.4$ and 360.0 in positive ion mode. SBA-15 could enrich most of the compounds (except BPS and PCP), but the intensities in negative ion mode were lower than those obtained with CMK-8 (Figure 3G,H). When using MCM-41, only a peak of CTAB was observed while peaks for other compounds were absent (Figure 3I,J). We also studied the enrichment performance of these materials in the presence of $10 \mu \mathrm{g} / \mathrm{mL} \mathrm{HA}$, and similar results were obtained (Supporting Information Figure S5). CMK-8 showed the best performance among the tested materials.

It can be recognized from the aforementioned results that the efficiency of enrichment was affected by the pore size and chemical nature of ordered mesoporous materials. The materials having wide pores (CMK-8 and SBA-15) were much more efficient than those having narrow pores (CMK-3 and MCM-41). Although narrow pore sizes could produce higher specific surface areas than wide pore size (Table 1 ), they might be easily blocked by large molecules, thereby hindering the intake of small molecules. Furthermore, the enrichment was closely dependent on the chemical nature of the materials. The hazardous compounds can interact with the enrichment materials via hydrophobic interaction, physical adsorption, or electrostatic interaction. Ordered mesoporous carbon (CMK8) worked better than ordered mesoporous silica (SBA-15), probably because mesoporous carbon is more hydrophobic than mesoporous silica ${ }^{7}$ and thus has a stronger affinity for lowmass hazardous compounds that consist of a large number of aromatic and hydrophobic functional groups. Accordingly, we demonstrate that CMK-8 is a highly efficient material for sizeselective enrichment of low-mass hazardous compounds, and it was used in the following experiments.

Validation of the Method with Real Samples. We validated this method with spiked real complex samples, including human serum, urine, and river water samples. Without enrichment no target compounds were detected in spiked serum samples (Figure 4A,B). After enrichment with CMK-8, all of the hazardous compounds could be detected (Figure 4C,D). The peak for TBBPA was weak, but high peak intensities were obtained for other compounds. The method has also been successfully applied to the analysis of spiked urine and river water samples (Supporting Information Figures S6 and S7). It should be noted that real samples often contain high concentrations of salts, which would cause serious ionization suppression effect in MS detection. The ability of direct analysis of highly saline samples without additional desalting steps indicates that the enrichment with CMK-8 can not only preconcentrate the target compounds and exclude the interferences from large molecules but also remove salts from samples due to its specific affinity for the hazardous compounds.

Analytical Performance. Table 2 lists detection limits (LODs) for the target analytes. The LODs for all of the compounds (except TBBPA and As ${ }^{\mathrm{III}}$ ) were at parts per trillion (ppt) or even sub-ppt levels. This sensitivity is sufficiently high that it enables direct screening and identification of most of the low-mass hazardous compounds in biological and environmental samples without any other enrichment or extraction procedures. Such a high sensitivity is ascribed to the combined use of ordered mesoporous carbon and graphene. The former provides highly efficient and selective enrichment, and the latter

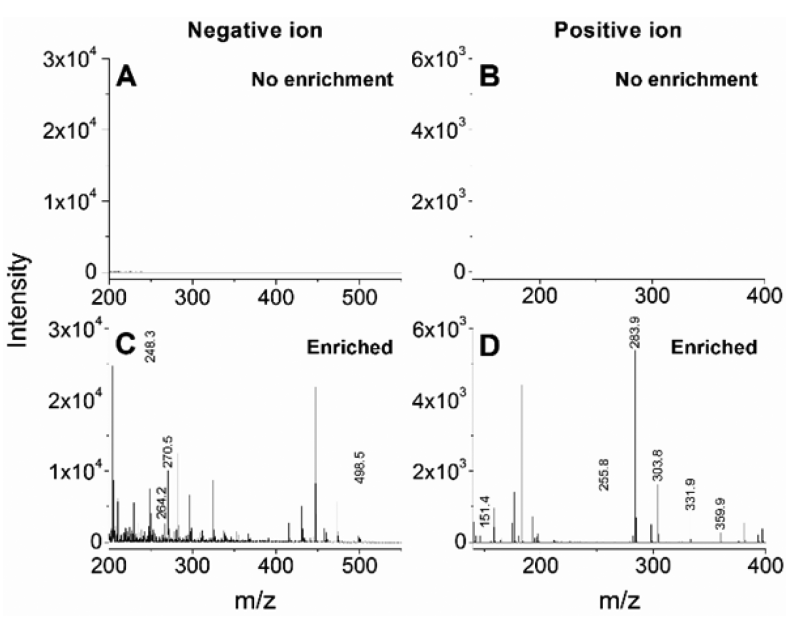

Figure 4. Analysis of spiked human serum samples. Direct analysis (A, B) without enrichment, and (C, D) with enrichment using CMK-8. The MALDI-TOF MS was performed in both negative ion (left column) and positive ion mode (right column). The analyte concentrations were the same as those stated in Figure 3. The $\mathrm{m} / \mathrm{z}$ values for the features peaks of the target compounds are labeled in the spectra.

improves the LDI efficiency and reduces the background noises in MS detection. We compare the sensitivity with that achieved with conventional methods such as GC or HPLC coupled to MS in terms of these compounds (see Supporting Information Table S2). For some compounds (e.g., BDE-47, PCP, and $\left.\mathrm{BAC}\left(\mathrm{C}_{16}-\mathrm{C}_{18}\right)\right)$, the sensitivity achieved in this study was better than or comparable to that obtained with conventional methods. ${ }^{19-21}$ For other compounds, this method yielded inferior sensitivity, but it could satisfy the demand for routine monitoring. For example, the upper limit for serum E2 for an adult male is $55 \mathrm{pg} / \mathrm{mL}^{22}$ An exception is As ${ }^{\mathrm{III}}$, as the "action level" for As set by the U.S. FDA is $10 \mathrm{ppb}^{23}$ and thus an extra enrichment step may be required for As ${ }^{\mathrm{III}}$. The reproducibility of the analysis was also satisfactory. The shot-to-shot relative standard deviations (RSDs) in MALDI were in the range of $6.2-15.1 \%$, and the extraction-to-extraction RSDs of the enrichment ranged from 13.8 to $18.3 \%$ (Supporting Information Table S3).

Another prominent advantage of this method is that it can simultaneously analyze multiple types of hazardous compounds in a single run, and thus one does not need to establish and optimize methods for individual compounds. Furthermore, the enrichment with CMK-8 is very facile and fast $(\sim 2 \mathrm{~h})$. Due to the inherent high-throughput analysis capability of MALDITOF MS, this method can cut much time for analysis.

Screening and Identification of Hazardous Chemicals in Human Sera. We applied this method to rapidly screen and identify low-mass hazardous compounds in human serum samples, which were collected from 5 healthy male athletes and 30 workers in a perfluorochemical plant. Figure 5A shows typical MS spectra for serum samples from athletes and workers. In athlete serum samples, no hazardous compounds were identified. However, several perfluorochemicals could be easily detected in all of the worker serum samples, some of which produced very high intensities $\left(>10^{4}\right.$; Supporting Information Table S4). Compared to athlete serum, we observed three new strong peaks in worker serum in negative ion mode at $m / z 298.4,398.6$, and 498.6 (Figure 5A). In addition to PFOS at $m / z$ 498.6, the peaks at $\mathrm{m} / z 298.4$ and 

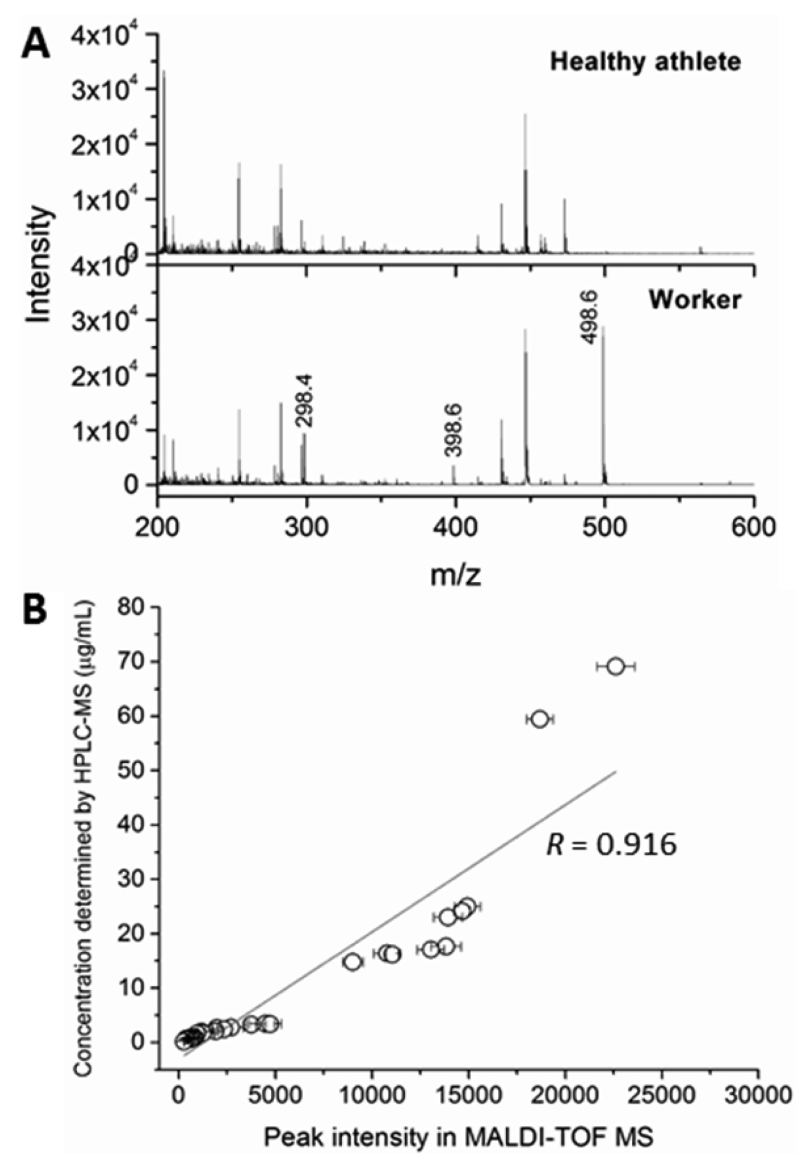

Figure 5. Screening and identification of perfluorochemicals in human serum samples from 30 workers in a perfluorochemical plant and 5 healthy male athletes. (A) Typical mass spectra for serum samples from workers and healthy athletes. (B) Comparison of screening results by MALDI-TOF MS and concentrations determined by HPLC-ESI-MS/MS. No PFOS could be detected in athlete sera, so the plot only contains results for the workers.

398.6 were identified to be $[\mathrm{M}-\mathrm{H}]^{-}$peaks of perfluorobutanesulfonic acid (PFBS) and perfluorohexanesulfonate (PFHxS), respectively. This result indicates that this method can be used to scan and identify unknown hazardous compounds in complex media.

To verify the results obtained by MALDI-TOF MS, we also measured the concentration of PFOS in these serum samples by HPLC-MS/MS. As plotted in Figure 5B, a linear correlation $(R=0.916)$ was found between the peak intensity in MALDITOF MS and the concentration obtained by HPLC-MS/MS. The serum concentration of PFOS of the workers was in the range of $0.186-69.1 \mu \mathrm{g} / \mathrm{mL}$ (Supporting Information Table S4). The good consistence in results from these two techniques demonstrates that this method is competent and reliable for rapid screening and semiquantitative analysis of trace low-mass compounds in complex samples. Furthermore, due to the high throughput and simplicity of this method, we could finish the analysis of 35 samples within 3-4 h; however, in the case of HPLC-MS/MS, complex sample preparation procedures such as liquid-liquid extraction and solid-phase extraction were required (see the Supporting Information for details) and it took over 3 days to finish the analysis of the same set of samples.

\section{CONCLUSIONS}

In summary, we have developed a new method for highthroughput and rapid screening and identification of low-mass hazardous compounds in complex media based on size-selective enrichment on ordered mesoporous carbon CMK-8 and MALDI-TOF MS with graphene as a matrix. This method does not require complicated sample preparation procedures and shows good tolerance for salt. The sensitivity for the typical compounds can reach ppt levels. It has been successfully applied to diverse complex matrices and to screen and identify hazardous compounds in human serum samples. With the advantages of high throughput, quickness, high sensitivity, and low cost, this method provides a promising tool for environmental health research and also keeps great potentials to be applied in omics (e.g., exposomics and metabonomics) studies. ${ }^{24-26}$

\section{ASSOCIATED CONTENT}

\section{Supporting Information}

Text describing the experimental details, tables listing MS intensities of typical low-mass compounds, comparison of LODs obtained with the present and previous methods, analysis reproducibility, and peak intensities and concentrations of PFOS from 30 workers and 5 healthy athletes, and figures showing $\mathrm{N}_{2}$ sorption isotherms and pore distribution curves of the ordered mesoporous materials, graphene TEM image, effects of BSA and HA on MS signals of typical low-mass compounds, comparison of different ordered mesoporous materials in extraction and enrichment of typical low-mass compounds, and analysis of spiked human urine samples and spiked river water samples. The Supporting Information is available free of charge on the ACS Publications website at DOI: 10.1021 /acs.analchem.5b01550.

\section{AUTHOR INFORMATION}

\section{Corresponding Author}

*E-mail: qianliu@rcees.ac.cn.

\section{Notes}

The authors declare no competing financial interest.

\section{ACKNOWLEDGMENTS}

This work was cosupported by the Strategic Priority Research Program of the Chinese Academy of Sciences (XDB14010400), the National Basic Research Program of China (2015CB931903 and 2015CB932003), the National Natural Science Foundation of China (21377141, 21422509, and 21321004), and the Excellent Young Scientist Grant of the Chinese Academy of Sciences.

\section{REFERENCES}

(1) Richardson, S. D.; Ternes, T. A. Anal. Chem. 2014, 86, 28132848.

(2) la Farre, M.; Perez, S.; Kantiani, L.; Barcelo, D. Trends Anal. Chem. 2008, 27, 991-1007.

(3) Richardson, S. D. Anal. Chem. 2012, 84, 747-778.

(4) Wakida, S.; Chiba, A.; Matsuda, T.; Fukushi, K.; Nakanishi, H.; Wu, X. L.; Nagai, H.; Kurosawa, S.; Takeda, S. Electrophoresis 2001, 22, 3505-3508.

(5) Wu, Z. X.; Zhao, D. Y. Chem. Commun. (Cambridge, U. K.) 2011, 47, 3332-3338

(6) Shi, Y. F.; Wan, Y.; Zhao, D. Y. Chem. Soc. Rev. 2011, 40, 38543878 . 
(7) Qin, H. Q.; Gao, P.; Wang, F. J.; Zhao, L.; Zhu, J.; Wang, A. Q.; Zhang, T.; Wu, R.; Zou, H. F. Angew. Chem., Int. Ed. 2011, 50, 1221812221.

(8) Sun, N. R.; Deng, C. H.; Li, Y.; Zhang, X. M. Anal. Chem. 2014, $86,2246-2250$.

(9) Teng, W.; Wu, Z. X.; Feng, D.; Fan, J. W.; Wang, J. X.; Wei, H.; Song, M. J.; Zhao, D. Y. Environ. Sci. Technol. 2013, 47, 8633-8641. (10) Sun, Z. K.; Deng, Y. H.; Wei, J.; Gu, D.; Tu, B.; Zhao, D. Y. Chem. Mater. 2011, 23, 2176-2184.

(11) Tian, R. J.; Zhang, H.; Ye, M. L.; Jiang, X. G.; Hu, L. H.; Li, X.; Bao, X. H.; Zou, H. F. Angew. Chem., Int. Ed. 2007, 46, 962-965.

(12) James, D. K.; Tour, J. M. Acc. Chem. Res. 2013, 46, 2307-2318.

(13) Allen, M. J.; Tung, V. C.; Kaner, R. B. Chem. Rev. 2010, 110, 132-145.

(14) Dong, X. L.; Cheng, J. S.; Li, J. H.; Wang, Y. S. Anal. Chem. 2010, 82, 6208-6214.

(15) Tang, L. A. L.; Wang, J. Z.; Loh, K. P. J. Am. Chem. Soc. 2010, 132, 10976-10977.

(16) Lu, M. H.; Lai, Y. Q.; Chen, G. N.; Cai, Z. W. Anal. Chem. 2011, $83,3161-3169$.

(17) Liu, Q.; Cheng, M. T.; Jiang, G. B. Chem.-Eur. J. 2013, 19, 5561-5565.

(18) Liu, Q.; Cheng, M. T.; Wang, J.; Jiang, G. B. Chem.-Eur. J. 2015, 21, 5594-5599.

(19) Bacaloni, A.; Callipo, L.; Corradini, E.; Giansanti, P.; Gubbiotti, R.; Samperi, R.; Laganà, A. J. Chromatogr. A 2009, 1216, 6400-6409.

(20) Zhou, Y.; Jiang, Q. W.; Peng, Q.; Xuan, D. L.; Qu, W. D. Chemosphere 2007, 70, 256-262.

(21) Ding, W.-H.; Liao, Y.-H. Anal. Chem. 2001, 73, 36-40.

(22) GPNotebook-Reference range (oestradiol); http://www. gpnotebook.co.uk/simplepage.cfm? ID =570818627\&linkID = 24801\&cook=yes (accessed Apr. 6, 2015).

(23) Supporting Document for Action Level for Arsenic in Apple Juice; U.S. Food and Drug Administration: Silver Spring, MD, USA, 2013.

(24) Lioy, P. J. Environ. Health Perspect. 2010, 118, 1081-1090.

(25) Wild, C. P.; Scalbert, A.; Herceg, Z. Environ. Mol. Mutagen. 2013, 54, 480-499.

(26) Athersuch, T. J. Bioanalysis 2012, 4, 2207-2212. 